\title{
Asociación de Clostridium difficile, su toxina A y el daño histopa- tológico en pacientes con diarrea nosocomial
}

\author{
Association of Clostridium difficile, its toxin A and histopathological dam- \\ age in patients with nosocomial diarrhea \\ Enrique Martin-Alva, Flora Chávez y Pedro Mercado
}

Departamento de Microbiología y Parasitología, Facultad de Ciencias Biológicas, Universidad Nacional de Trujillo, Ciudad Universitaria, Av Juan Pablo II S/N. Trujillo, Perú.

Email: Enrique Martin Alva enrique.martinalva@gmail.com

Presentado: $\quad 06 / 09 / 2006$ Aceptado: $\quad 11 / 08 / 2007$

\section{Resumen}

Esta Investigación estuvo orientada a determinar la asociación entre la presencia de Clostridium difficile, su toxina A y el daño histopatológico, en pacientes del Servicio de Gastroenterología del Hospital Victor Lazarte Echegaray entre enero y agosto del 2003. La población de estudio estuvo conformada por todos los pacientes (24) admitidos para su tratamiento de diarreas postantibióticas intrahospitalarias. De cada paciente se recolectaron heces para el diagnóstico de $C$. difficile, así como, para determinar la presencia de su toxina A, mediante un Kit comercial (Oxoid). En los mismos pacientes, se hizo la evaluación del daño histopatológico, con el apoyo del especialista del Hospital; tipificando el daño como Lesión tipo I, II o III. Los resultados obtenidos indican que 18 pacientes $(75 \%)$ tienen $C$. difficile, pero sólo $15(62,5 \%)$ de ellos tuvieron toxina $A$, habiendo tres $(12,5 \%)$ sin lesión colónica además, predominó la lesión tipo I (37,5\%). De nueve pacientes sin toxina $A$, uno $(4,16 \%)$ tuvo $C$. difficile y presentó lesión colónica, mientras que dos pacientes $(8,33 \%)$ no tuvieron toxina A y sin $C$. difficile y con lesión colónica tipo I. Además se encontró asociación significativa entre este $C$. difficile y su toxina, pero no de estas con el tipo de lesión.

Palabras clave: Diarrea asociada a antibióticos, Clotridium difficile, daño histopatológico, toxina A, colitis seudomembranosa.

\section{Abstract}

This study determines the association of Clotridium difficile and its toxin A with the histopathological damage in patients from the Gastroenterology Service of Victor Lazarte Echegaray Hospital, TrujilloPerú from january to august, 2003. The study was done in 24 patients admitted with treatment of nosocomial diarrhea. Stool samples were colleted from each patient to diagnose $C$. difficile and its toxin A using a commercial kit (Oxoid). The evaluation histological of damage was made in each patients, the damage were typified in three types of lesions (I, II or III). It was found that $18(75 \%)$ patients had $C$. difficile, but only $15(62,5 \%)$ of them had toxin A in faeces and three $(12,5 \%)$ did not have colonic lesion. The lesion type I, was the most predominant (37,5\%). From nine patients without toxin $A$ in faeces, one $(4,16 \%)$ had $C$. diffficile and colonic lesion, by the other hand, two $(8,33 \%)$ patients did not have toxin $A$ and $C$. difficile but they had colonic lesion type I. It was not association between $C$. difficile and its toxin $A$. There is an important increase of $C$. difficile incidence. The presence of patients with $C$. difficile but without toxin $A$ is explained by the existence of atoxigenic strains, being the lesions produce by several causes, not only by the presence of toxins.

Keywords: Antibiotic associated diarrhoea, Clotridium difficile, histopathological damage, toxin A, pseudomembranous cholitis.

\section{Introduction}

En los últimos años se ha registrado un importante incremento en la incidencia de infección intestinal por Clostridiun difficile. En un estudio realizado en el Reino Unido se reveló como el primer patógeno intestinal en pacientes ancianos hospitalizados, por delante de clásicos agentes de diarrea, Salmonella y Campylobacter (Djuretic et al., 1996). El aumento de casos reportados puede ser debido en parte a una mejora en el diagnóstico y a una mayor comunicación de los casos, pero indudablemente también refleja un aumento real en la magnitud del problema (Johnson y Gerding, 1996).

Estudios prospectivos revelan que hasta un $21 \%$ de los pacientes con cultivo negativo en el momento del ingreso hospitalario son colonizados por $C$. difficile durante la estancia La mayoría de estos enfermos ingresados e infectados por este microorganismo se mantienen asintomáticos. Sin embargo, se ha estimado que hasta $1 \%$ de los pacientes ingresados, tratados con antibióticos, podrían presentar diarrea secundaria por infección con C. difficile (Johnson y Gerding, 1996).
Desde la fuente de infección o desde el reservorio a través de diversos mecanismos de transmisión, $C$. difficile alcanza al huésped potencialmente susceptible. Una vez en el colon, las esporas de $C$. difficile adoptan la forma vegetativa, se multiplican y las cepas toxigénicas pueden producir dos tipos de exotoxinas; la toxina A con efectos fundamentalmente enterotóxicos en animales (Chávez-Olarte et al., 1997; Cheng et al., 1997). La citoxina $\mathrm{B}$, por su parte, carece de efectos enterotóxicos en animales. Por este motivo se le había atribuido un escaso valor patogénico en la producción de colitis. Sin embargo, un estudio reciente evidencia que in vitro, se comporta también como una potente enterotoxina necrosante, con un poder 10 veces superior a la toxina A para dañar la mucosa colónica humana (Djurteic et al. 1996; Depitre et al., 1993).

En el laboratorio, el método ideal de diagnóstico de la infección por $C$. difficile es la detección de la toxina $\mathrm{B}$ en las heces del enfermo dada su elevada sensibilidad y especificidad. Este test persigue no sólo la demostración de la presencia de $C$. difficile, sino también, determinar si se trata de una cepa toxigénica; y consiste en la verificación de los efectos citopáticos de esta toxina 
en los cultivos de fibroblastos humanos in vitro, neutralizados al añadir la antitoxina de $C$. sordelii. Su resultado se expresa como positivo o negativo dado que no parece existir correlación entre gravedad clínica y concentraciones de toxina. Desafortunadamente, presenta varios inconvenientes, tales como su crecimiento lento (requiere 48-72 h), el elevado costo y complejidad en el cultivo, requiriendo el mantenimiento de líneas celulares, técnica que no está al alcance de todos los laboratorios (Pothoulakis et al., 1988; Pothoulakis y Lamont, 1991)

Las lesiones del colon producidos por $C$. difficile pueden presentar en algunos casos áreas con necrosis epitelial en parches, ulceración epitelial, y en otros llegar hasta la necrosis epitelial difusa cubierta por una seudomembrana formada por polimorfonucleares. La curación clínica del proceso suele ir acompañada de la resolución histológica, persistiendo sólo cierta irregularidad en la arquitectura glandular (Portillo et al., 2002).

En las últimas décadas, ha habido un incremento en la incidencia de la infección intestinal por $C$. difficile, expresándose en diferentes formas, desde brotes de diarrea hasta colitis pseudomenbranosa. Sin embargo, en el contexto nacional y local, no se han establecido modelos de estudio sistemático para confirmar esta tendencia epidemiológica y que a la vez permita conocer mejor la relación del patógeno con el huésped, lo cual permitiría establecer medidas de control compatibles con nuestra realidad, medidas que fluctúan desde la política antimicrobiana, detección temprana, enfermos portadores, etc.

El presente estudio, estuvo orientado a determinar si existe asociación entre la presencia de Clostridium difficile, su toxina A y el daño histopatológico, en pacientes con diarrea nosocomial atendidos en el Servicio de Gastroenterología del Hospital Víctor Lazarte Echegaray de Trujillo - Perú, durante el año 2003.

\section{Material y métodos \\ Población}

El universo muestral y la muestra estuvieron conformados por todos los pacientes admitidos para su tratamiento de diarreas postantibióticas intrahospitalarias, atendidos en el Servicio de Gastroenterología del Hospital Víctor Lazarte Echegaray, EsSalud Trujillo, durante el período de enero a agosto del 2003 y que cumplian con los criterios de inclusión (pacientes con diarrea postantibiótica intrahospitalaria y pacientes con un año a más de edad) y exclusión (Pacientes que habían recibido tratamiento con Vancomicina y Metronidazol, VIH positivos, menores de un ańo y que al ingresar al hospital presentaron diarrea).

\section{Toma de muestra y conservación}

Las heces fueron recogidas en recipientes adecuados y transportadas de inmediato al Laboratorio de Fisiología y Genética

Tabla 1. Relación de Clostridium difficile y su toxina A en heces de 24 pacientes del Servicio de Gastroenterología del Hospital Víctor Lazarte Echegaray, enero-agosto del 2003.

\begin{tabular}{cccccccc}
\hline & \multicolumn{3}{c}{ Toxina A } & \multicolumn{2}{c}{ Total } \\
\cline { 2 - 5 } & \multicolumn{2}{c}{ Presente } & \multicolumn{2}{c}{ Ausente } & & \multicolumn{2}{c}{ To } \\
\hline Cultivo & $\mathrm{fi}$ & $\%$ & $\mathrm{fi}$ & $\%$ & & $\%$ \\
$\mathrm{CD}+{ }^{*}$ & 15 & 62,50 & 03 & 12,50 & & 18 & 75 \\
$\mathrm{CD}{ }^{*}$ & 00 & 00 & 06 & 25,00 & & 06 & 25 \\
\hline
\end{tabular}

${ }^{*} \mathrm{CD}+=$ presencia de $C$. difficile en el cultivo;

CD - = ausencia de $C$. difficile en el cultivo
Tabla 2. Relación entre la presencia de Clostridium difficile, su toxina A con el tipo de lesión en 15 pacientes del Servicio de Gastroenterología del Hospital Víctor Lazarte Echegaray, enero-agosto del 2003.

\begin{tabular}{cccc}
\hline & \multicolumn{3}{c}{ Toxina positiva (+) } \\
\cline { 2 - 4 } Tipo de lesión & \multicolumn{3}{c}{ Cultivo } \\
\cline { 2 - 4 } & & + & - \\
\hline Sin lesión & $\mathrm{fi}$ & 3 & 0 \\
& $\%$ & 12,5 & 0 \\
Tipo I & $\mathrm{fi}$ & 9 & 0 \\
& $\%$ & 37,5 & 0 \\
\multirow{2}{*}{ Tipo II } & $\mathrm{fi}$ & 1 & 0 \\
& $\%$ & 4,17 & 0 \\
Tipo III & $\mathrm{fi}$ & 2 & 0 \\
& $\%$ & 8,33 & 0 \\
\hline TOTAL & $\mathrm{fi}$ & 15 & 0 \\
& $\%$ & 62,5 & 0 \\
\hline
\end{tabular}

Bacteriana de la Universidad Nacional de Trujillo, de cada muestra se tomaron $5 \mathrm{~g}$ que sirvieron para el aislamiento de $C$. difficile y detección de su toxina $\mathrm{A}$, mediante el método de inmunodifusión. La muestra de biopsia fue tomada según criterio del especialista, para su posterior evaluación.

\section{Identificación y Detección de la toxina A de Clos- tridium difficile:}

Para la determinación de $C$. difficile se tuvo en cuenta las características morfoculturales y la tipificación bioquímica. Mientras que la detección de la toxina se hizo de acuerdo a lo especificado en el Toxin Detection Kits (Oxoid) para C. difficile Toxin A Test (Lenette et al., 1987).

\section{Evaluación del daño histopatológico}

Los resultados de las biopsias colónonicas fueron categorizadas en tres tipos de daños:

Tipo I: Se pueden apreciar acumulaciones focales de polimorfonucleares con áreas de necrosis epitelial parcheada y exudación de fibrina y neutrófilos en la luz colónica.

Tipo II: presenta un exudado más prominente que se origina de un área de ulceración epitelial, manteniéndose intacta la mucosa circundante.

Tipo III: Consiste en la necrosis epitelial difusa (no focal como sucedía en los tipos anteriores), cubierta por una pseudomembrana formada por polimorfonucleares, fibrina y detritus celulares.

\section{Análisis Estadístico}

Tablas de frecuencia y de contingencia de doble entrada fueron elaboradas con los datos obtenidos, aplicándose la prueba de Ji Cuadrado, para estimar la asociación entre variables; probándose su significancia a un nivel de confianza del 95\%. Se emplearon los programas estadísticos de Microstat y SSPS.

\section{Resultados}

De los 24 pacientes evaluados, 15 de ellos $(62,5 \%)$ presentaron toxina A de $C$. difficile en heces y nueve $(37,5 \%)$ no presentaron esta toxina. Los 15 pacientes con toxina A positiva, tuvieron cul- 
Tabla 3. Relación entre la presencia de Clostridium difficile, y la ausencia de su toxina $A$ con el tipo de lesión en nueve pacientes del Servicio de Gastroenterología del Hospital Víctor Lazarte Echegaray, enero-agosto del 2003.

\begin{tabular}{ccccc}
\hline & \multicolumn{3}{c}{ Toxina negativa (- ) } & \\
\cline { 2 - 4 } Lesión & \multicolumn{3}{c}{ Cultivo } & Total \\
\cline { 2 - 4 } Sin lesión & $\mathrm{fi}$ & 1 & 4 & 5 \\
& $\%$ & 4,16 & 16,67 & 20,83 \\
\multirow{2}{*}{ Tipo I } & $\mathrm{fi}$ & 1 & 2 & 3 \\
& $\%$ & 4,16 & 8,33 & 12,50 \\
\multirow{2}{*}{ Tipo II } & $\mathrm{fi}$ & 1 & 0 & 1 \\
& $\%$ & 4,16 & 0 & 4,16 \\
\multirow{3}{*}{ Tipo III } & $\mathrm{fi}$ & 0 & 0 & 0 \\
& $\%$ & 0 & 0 & 0 \\
\hline \multirow{2}{*}{ Total } & $\mathrm{fi}$ & 3 & 6 & 9 \\
& $\%$ & 12,5 & 25,0 & 37,5 \\
\hline
\end{tabular}

tivo positivo para $C$. difficile; mientras que de los nueve pacientes con toxina A negativa sólo seis (25\%), presentaron ausencia de este en el cultivo y los tres restantes (12,5\%) manifestaron presencia de $C$. diffcile. Además se observa que, de los 24 pacientes evaluados, 18 de ellos (75\%) presentaron presencia de $C$. difficile en el cultivo de heces (Tabla 1).

De los 15 pacientes con toxina A positiva en heces y presencia de $C$. difficile en el cultivo, tres (12,5\%) de ellos no manifiestaron ningún tipo de lesión colónica; teniendo la mayoría de ellos, nueve pacientes (37,5\%), lesión tipo I. Sólo un paciente tuvo lesión tipo II y dos pacientes mostraron lesión tipo III (Tabla 2).

De los nueve $(37,5 \%)$ pacientes que no presentaron toxina $\mathrm{A}$ de $C$. difficile en heces, tres (12,5\%) de ellos dieron cultivo positivo de este microorganismo, de los cuales uno careció de lesión colónica, otro presentó lesión tipo I y el otro mostró lesión tipo II. A su vez, de los seis $(25 \%)$ pacientes con cultivo negativo a $C$. difficile, cuatro $(16,67 \%)$ no presentaron lesión y dos $(8,33 \%)$ tuvieron lesión colónica tipo I. Por otro lado, de nueve (37,50\%) pacientes que no presentaron toxina A, cinco $(20,83 \%)$ no presentaron lesión colónica; tres $(12,50 \%)$ tuvieron lesión tipo I y uno $(4,16 \%)$ presentó lesión tipo II (Tabla 3.)

$\mathrm{Al}$ relacionar la variable cultivo de heces (presencia o ausencia de $C$. difficile) con la toxina A (presente o ausente), se determinó que existe asociación entre estas variables; es decir, que la presencia de toxina $\mathrm{A}$, tiene relación directa con la presencia de este microorganismo $\left(\mathrm{Xi}^{2} \mathrm{c}=13,33 ; \mathrm{Xi}^{2} \mathrm{tab}=3,84 ; \mathrm{P}<0,05\right)$. Así mismo, luego de la correspondiente prueba de hipótesis, se detectó que no existe asociación entre el tipo de lesión y la presencia de toxina $\mathrm{A}$, a un nivel de confianza del $95 \%\left(\mathrm{Xi}^{2} \mathrm{c}=\right.$ 4,266; $\left.\mathrm{Xi}^{2} \mathrm{tab}=7,81 ; \mathrm{P}<0,05\right)$ y al relacionar la presencia o no de $C$. difficile con la lesión colónica, luego de la correspondiente prueba de hipótesis, no existe asociación entre estas variables, aun nivel de confianza del $95 \%$; es decir que el tipo de lesión no depende de la presencia de $C$. difficile $\left(\mathrm{Xi}^{2} \mathrm{c}=4,44 ; \mathrm{Xi}^{2} \mathrm{tab}=\right.$ 7,81; $\mathrm{P}<0,05)$.

\section{Discusión}

Los resultados indican que el $75 \%$ de la población evaluada sufría del problema de diarreas postantibióticas, lo cual consti- tuye un elevado porcentaje de morbilidad, si se compara con los tenores mundiales reportados, que sólo tienen entre el 15 a $20 \%$ como máximo claramente asociado con este germen (Fekety, 1997).

De los 24 pacientes evaluados (Tabla 1), 18 (75\%), presentaron $C$. difficile en el cultivo de heces y sólo 15 (62,50\%) mostraron presencia de toxina A; de donde se desprende que en tres pacientes, a pesar haber presentado $C$. difficile en las heces, no fue detectada la producción de la toxina A. Esto se explica porque existen casos de colitis seudomembranosa producida por cepas no toxigénicas, además de la existencia de un número importante de portadores asintomáticos, en los que se detectan cepas toxigénicas comportándose como saprófitos; lo cual sugiere la existencia de otros mecanismos patogénicos desconocidos (Pothoulakis y Lamont, 1993). En este sentido, factores dependientes del huésped, fundamentalmente inmunitarios, no bien estudiados, podrían ser cruciales modulando la expresión clínica. Cheng et al. (1997) señalan que la presencia o ausencia de toxinas, o la ribotipificación de las cepas, se asociaba con la repercusión clínica, que parecía ser determinada más bien por las circunstancias del huésped: cuantas más enfermedades asociadas y cuanto mayor la duración del tratamiento antibiótico, mayor la repercusión de la infección.

Así mismo, de los 15 pacientes con toxina A (Tabla 2), nueve $(37,5 \%)$, tuvieron lesión tipo I y sólo tres carecieron de lesión; mientras que dos de ellos tuvieron lesión tipo III. Al respecto, es importante tener en cuenta que, si bien la presencia de las típicas seudomembranas es prácticamente patognomónica de la enfermedad, su ausencia no excluye en absoluto el diagnóstico de diarrea asociada a $C$. difficile, ya que son frecuentes otros patrones endoscópicos, en relación a los diferentes grados de severidad clínica, que oscilan desde la existencia de una mucosa totalmente normal hasta otras formas de colitis sin formación de seudomembranas. En los casos clínicamente leves la mucosa suele ser normal o con mínimos signos inflamatorios no específicos. En casos más severos puede observarse eritema, edema, friabilidad y úlceras que ocasionalmente pueden hacer difícil el diagnóstico diferencial con la colitis ulcerosa, incluso en el estudio anatomopatológico (Price y Davies, 1977).

Lo expresado en párrafos anteriores explicaría la presencia de lesiones colónicas en pacientes con $C$. difficile, pero con ausencia de toxina A. Así mismo, la presencia de lesiones en pacientes sin este microorganismo y sin su toxina $A$, puede ser explicada por la presencia de otras infecciones y patologías no infecciosas y sin causa conocida. Al respecto, existen algunos casos publicados de colitis seudomembranosa, secundarios a otras etiologías. Así, otras infecciones producidas por Shigella, E. coli, Plesiomonas, hongos, citomegalovirus y otros procesos de naturaleza no infecciosa como la enfermedad inflamatoria intestinal, la isquemia o fármacos como el oro y el diclofenaco pueden ser origen de colitis con formación de seudomembranas. Estos procesos deben ser tenidos en cuenta, sobre todo en ciertos contextos clínicos, en el diagnóstico diferencial de la colitis seudomembranosa.

La presencia de toxina A y de $C$. difficile, manifiestan, estadísticamente, estar asociadas; lo cual podría ser explicado porque es más frecuente encontrar cepas de esta bacteria toxigénicas que atoxigénicas. Por otro lado, la ausencia de asociación entre presencia de toxina A o de $C$. difficile con el tipo de lesión, ratifica lo expresado anteriormente; es decir que hay cepas de $C$. difficile, 
tanto toxigénicas como no también; o que las lesiones colónicas pueden deberse a otros agentes infecciosos u otras patologías no conocidas (Pothoulakis y Lamont, 1993).

\section{Literatura citada}

Bouza E., P. Padilla, P. Catalán, C. Sánchez-Carrillo, R. Blázquez \& T. Peláez. 1996. Diarrea asociada a Clostridium difficile: experiencia durante un año en un hospital general. Rev. Clin. Esp. 196:424-430.

Chávez-Olarte E., M. Weidman, C. Eichel-Streiber \& M. Thelestam. 1997. Toxins A and B from Clostridium difficile differ with respect to enzymatic potencies, celular substrate specificities and surface binding to culturated cells. J. Clin Invest. 100: $1734-41$

Cheng, S.H.; J.J. Lu; T.G. Young; C.L. Perng \& W.M. Nhin. 1997. Clostridium difficile-associated diseases: comparison of symptomatic infection versous carriage on the basis of risk factors, toxin production and genotyping results. Clin. Infec. Dis. 25: 157-158.

Depitre C., M. Delmée \& V. Avesani. 1993. Serogroup F strains of Clostridium difficile produce toxin B but not toxin A. J. Med. Microbiol. 38:434-41.

Djuretic T, M.J. Ryan, D.M. Fleming \& P.G. Whall. 1996. Infectious intestinal disease in elderly people. Commun. Dis. Rev. CDR Rev. 6: 107-112
Fekety R. 1997. Guidelines for the diagnosis and management of Clostridium difficile-associated diarrhea and colitis. Am. J. Gastroenterol. 92: 739-750.

Johnson S. \& D. Gerding. 1996. Clostridium difficile. En: Mayhall CG, editor. Hospital epidemiology and infection control. Baltimore, Williams and Wilkins. Pp. 399-408

Lennette E., A. Ballows, W. Hauster \& H. Shadomy. 1987. Manual de Microbiología Clínica. 4ta Edic. Edit. Medica Panamericana. Bs As. Argentina. Pp 547-52

Portillo M., M. Castellanos, E. Cortés \& Chipnit R. 2002. Infeccion por Clostridium difficile. Gac.Med.Mex.22:623-637

Pothoulakis C. \& J.T. Lamont. 1991. Characterization of rabbit ileal receptors for Clostridium difficile toxin A: evidence for a receptor-coupled G protein. J.Clin.Invest.88:119-125.

Pothoulakis, C. \& J.T. Lamont. 1993. Clostridium difficile colitis and diarrhea. Gastroenterol. Clin. Am. 22: 623-637.

Pothoulakis, C.; R. Sullivan \& D.A. Meloick. 1988. Clostridium difficile toxin A stimulates intracellular calcium release and chemotactic response in human granulocytes. J. Clin. Invest. 81: 1741-1745.

Price, A.U. \& D.R. Davies. 1977. Pseudomembranous colitis. J Clin Pathol. 30: 112 . 